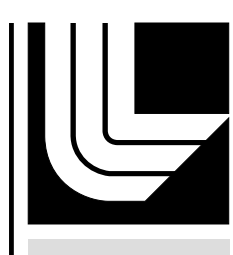

LAWRENCE LIVERMORE N A T IO N A L LABORATORY

\title{
Study of High Mach Number Laser Driven Blast Waves
}

A. Edens, T. Ditmire, J. F. Hansen, M. J. Edwards, R. G. Adams, P. Rambo, L. Ruggles, I. C. Smith, J. L. Porter

March 3, 2004

Physics of Plasmas 
This document was prepared as an account of work sponsored by an agency of the United States Government. Neither the United States Government nor the University of California nor any of their employees, makes any warranty, express or implied, or assumes any legal liability or responsibility for the accuracy, completeness, or usefulness of any information, apparatus, product, or process disclosed, or represents that its use would not infringe privately owned rights. Reference herein to any specific commercial product, process, or service by trade name, trademark, manufacturer, or otherwise, does not necessarily constitute or imply its endorsement, recommendation, or favoring by the United States Government or the University of California. The views and opinions of authors expressed herein do not necessarily state or reflect those of the United States Government or the University of California, and shall not be used for advertising or product endorsement purposes. 
UCRL-JRNL-202693

\title{
Study of High Mach Number Laser Driven Blast Waves
}

\author{
A. Edens and T. Ditmire \\ Department of Physics, University of Texas at Austin, Austin, TX 78712 \\ J. F. Hansen and M. J. Edwards \\ Lawrence Livermore National Laboratory, Livermore, CA, 94550 \\ R.G. Adams, P. Rambo, L. Ruggles, I.C. Smith, and J. L. Porter \\ Sandia National Laboratories, Albuquerque, NM, 87059
}

The study of blast waves produced by intense lasers in gases is motivated by the desire to explore astrophysically relevant hydrodynamic phenomena in the laboratory. We have performed a systematic scan of laser produced blast waves and have examined the blast wave structure over a wide range of drive laser energy. Lasers with energies ranging from $10 \mathrm{~J}-1000 \mathrm{~J}$ illuminated a pin target in either xenon or nitrogen gas, creating a spherical blast wave. We observe a strongly radiating blast wave in xenon gas while blast waves in nitrogen more closely approximate a pure Taylor-Sedov wave. We also find that at all laser energies, blast waves traveling through xenon gas had their hydrodynamic evolution significantly affected by the passage of the illumination laser. 


\section{Introduction}

The stabilty of blast waves has been a long standing problem of interest in the physics and astronomy communities ${ }^{1,2}$. In particular, there are several instabilities associated with the formation and evolution of supernova remnanta (SNRs) $)^{2-4}$. One important hydrodynamic feature, an overstability proposed by Vishniac et al. about 20 years ago ${ }^{5}$, arises from a mismatch between the ram and thermal pressures at the surface of a blast wave which can cause a growing, oscillating ripple on that surface. The growth rate of the overstability depends mainly on two factors, the wavelength of the perturbation and the thickness of the blast wave, which is determined by the gas' adiabatic index ${ }^{5}$. Energy losses through radiation make a strongly radiating blast wave cooler and thusly more compressible, leading to a thinner blast wave which is susceptible to the Vishniac overstability. Therefore the Vishniac overstability may be responsible for the large scale structure seen in some radiative SNRs ${ }^{2,6}$, and may also play a role in the formation of stars 3,7 .

There have been various experimental and computation studies attempting to confirm the existence and growth rates of the Vishniac overstability. Blondin et al. conducted a series of 1-D and 2-D radiative hydro simulations which appeared to confirm the existence of the overstability ${ }^{6}$. MacLow and Norman conducted 2-D simulations where they confirmed the theoretical growth rates for the overstability assuming the amplitude of the perturbation does not get large compared to the radius of the blast wave $^{8}$. In addition, over the previous fifteen years there have been a number of experimental attempts to observe the overstability ${ }^{9-11}$. These experiments have produced their blast waves chiefly through the creation of an explosion by irradiation of a target by 
a high-powered laser pulse. In these laser experiments the laser delivers a large quantity of energy in a small focal spot over a time period short compared to the evolution time of the resulting blast wave. A short intense point explosion is also how one would describe a supernova on astrophysical scales, making laser driven explosions well suited to studying similar physics.

Important early experimental work was published by Grun et al. ${ }^{9}$, using the Pharos III laser at the Naval Research Laboratory. They conducted experiments on the evolution of hemispherical blast waves produced in a background gas by single-sided irradiation of a foil by 200J laser pulses. Grun et al. reported observing the Vishniac overstability grow from noise in blast waves traveling through xenon gas back upstream of the incoming laser, while those blast waves traveling through nitrogen gas remained stable and the shock front remained smooth. However, whereas theory predicted a maximum growth rate going as $\mathrm{t}^{0.7}$ at an $\_$number of $\sim 50$, Grun et al. observed ${ }^{9}$ growth going as $\mathrm{t}^{1.6}$ at an _ number of $\sim 10$.

More recent work on cylindrical laser produced blast waves in Xe driven by an ultra-fast laser was published by Edwards et al. ${ }^{10}$. They observed evidence of the formation of a radiative shock but did not observe any perturbation growth on the shock front. The authors postulated the reason they saw no growth was that the Mach number of their blast wave was too low and that the wave was heated by efficient electron conduction between the hot core and the blast wave shell, leading to a lower effective adiabatic index and a thicker blast front. Theoretical work by Lamming et. al. ${ }^{12}$ seemed to confirm this idea by showing that there is a velocity cutoff for the overstability to affect blast waves traveling through xenon gas. However, Lamming et al. could not 
explain the discrepancy between theory and the NRL experiments in regards to the position and size of the maximal growth feature.

The velocity and temperature of a laser driven blast wave will increase with the increasing drive laser energy. Therefore, a systematic scan of laser energies should allow for a more comprehensive study of the overstability. In this letter we report on such a systematic examination of spherical blast waves produced by laser energies spanning two orders of magnitude, from $10 \mathrm{~J}$ to $1000 \mathrm{~J}$. This scan produced blast waves in both nitrogen and xenon including blast waves in xenon both above and below the $25 \mathrm{~km} / \mathrm{s}$ threshold for overstability growth predicted by Lamming et al ${ }^{12}$. We compare the results with theoretical simulations and the previous experimental work and find that laser preionization of the surrounding gas can significantly affect the dynamics and morphology of these shock experiments. The effect is particularly pronounced for blast waves traveling through xenon gas.

\section{Experimental Setup}

A schematic of our experimental setup is shown in Fig. 1. A pump laser varying in energy from 10-1000J was focused into a target chamber filled with between 5 and 10 Torr of xenon or nitrogen gas. The laser illuminated one side of a $\sim 0.5 \mathrm{~mm}$ pin target of steel or nylon. This created an explosion that formed a blast wave in the surrounding gas. The use of a pin target (as opposed to the planar foil targets used in Grun's experiment ${ }^{9}$ ) allowed us to examine the evolution of the blast wave both in the gas previously traversed by the laser on the front side of the pin and in the pristine gas on the back side of the pin. Some variable time later a probe laser pulse was fired. This passed through 
our experimental region and was then imaged with a dark-field imaging telescope. This diagnostic, (illustrated in the inset of figure 1) is sensitive to gradients in plasma density and is therefore well suited to examining the interface region between the ionized blast wave shell and unionized gas. We characterized the shock radius and front morphology as the blast wave expanded by scanning the time delay between the pump and probe beams.

Our experiments were performed on two different laser systems. The first was the Janus laser at Lawrence Livermore National Laboratory ${ }^{13}$. This laser fired 1ns pulses with pulse energies between $10 \mathrm{~J}$ and $150 \mathrm{~J}$ at $1053 \mathrm{~nm}$. Here a $5 \mathrm{~mJ}, 532 \mathrm{~nm}$ beam gated to $2 \mathrm{~ns}$ probed the blast wave. We performed higher laser drive energy experiments on the Z-Beamlet laser at Sandia National Laboratories ${ }^{14}$. This laser fired 1 ns pulses, with 527 $\mathrm{nm}$ wavelength at energies between $500 \mathrm{~J}$ and $1000 \mathrm{~J}$. The probe beam on these experiments was a $\sim 150 \mathrm{ps}$ beam with $10 \mathrm{~s}$ of $\mathrm{mJ}$ at $1064 \mathrm{~nm}$.

\section{Data and Analysis}

We have evidence that shock waves in Xe were strongly radiative at early times for the highest energy shots we conducted. First we observe a radiative precursor in front of the blast wave. This precursor takes the form of a "glow" ahead of the shock wave in the data reproduced in Figure 3 (described below). This glow was confirmed through the use of interferometry in which a region of increasing electron density was observed preceding the shock front. This precursor was seen for blast waves traveling through xenon at all energies, but in nitrogen the precursor was only observed for the highest laser energy shots. For the highest energy blast waves in xenon, the precursor extended off the 
field of view of the camera, which meant the precursor extended for several centimeters in all directions around the wave. However, this precursor wave only demonstrated that there was radiation coming from the blast waves, not that it was affecting the dynamics of the wave's evolution. To gauge the impact of the radiation we looked at the blast wave trajectories.

A blast wave can be characterized by its asymptotic trajectory $R(t)=\beta t^{\alpha}$ where the deceleration parameter $\alpha=V t / R$. Here $V$ and $R$ are the velocity and radius of the shock wave at time $t$ after the explosion. In a classical spherical, energy conserving Sedov-Taylor wave consisting of a high pressure low density central core pushing a thin compressed shell, $\alpha=2 / 5^{15}$. If the surrounding gas is sufficiently dense and radiative cooling is important, the deceleration parameter falls below the Taylor Sedov value and the rate of deceleration is faster than a purely energy conserving wave. When the thin shell of the blast wave is cooled efficiently by radiative losses $\alpha$ approaches $2 / 7$. In this so-called "pressure driven snowplow" regime the low density central core continues to exert pressure on a shell which cannot support itself and collapses to high density ${ }^{16}$. If radiative cooling of the hot low density core is also efficient, the pressure of the core becomes negligible, and the wave enters the "momentum conserving snow-plow" regime, in which $\alpha=1 / 4^{16}$. We measured the radial trajectory of blast waves in both Xe and $\mathrm{N}_{2}$ at all laser energies. The data for the 1000J shots are plotted in figure 2 . We see that at late times, both Xe and $\mathrm{N}_{2}$ blast waves follow the Taylor Sedov $\mathrm{t}^{2 / 5}$ trajectory. This is consistent with the trajectories observed by Grun et $\mathrm{al}^{9}$. However, at early time $(\mathrm{t}<200 \mathrm{~ns})$ the blast wave in Xe follows a trajectory with $\alpha=1 / 4$. This lower $\alpha$ seems to suggest that the blast waves in our experiments go through a strongly radiative stage prior to $200 \mathrm{~ns}$. 
Images of shockwaves traveling through 5-10 Torr of Xenon gas are shown on the left side of Fig. 3. The shockwaves were produced by a range of laser driver energies, $10 \mathrm{~J}, 500 \mathrm{~J}$, and 1000J. In all cases the laser pulse came in from the left of the picture. All show similar behavior. There is a marked difference between the section of blast wave traveling over the gas region traversed by the laser and the rest of the blast wave on the back side of the pin. The difference is a turbulent region with a large perturbation of roughly wave number 10 on the laser side of the wave that is not present on the back side of the wave. This feature is consistent in position and wave number with the Grun results ${ }^{9}$. The turbulent feature we see becomes increasingly pronounced with increasing energy, but the qualitative behavior is similar. However, there are smaller scale perturbations, closer in wave number to the theoretical maximal growth rate region, that appear only at higher energies. Though it is difficult to measure a growth rate for these perturbations due to their small size, they seem more consistent with the Vishniac overstability and are seen both in our higher energy data and in the data from Grun et al ${ }^{9}$. The xenon images can be contrasted with the images of blast waves traveling through 510 Torr nitrogen gas, shown on the right side of the same figure. Again we see blast waves produced by the same three laser drive energies. In this case the feature seen in xenon in the region traversed by the laser is greatly reduced and the small scale perturbations never arise. The fact that a qualitatively energy independent feature is seen only on blast waves traveling through Xenon and only on the side of the blast wave that interacted with the laser suggests that there is some effect of the laser's passage on the evolution of blast waves in xenon. This feature is evident both at low and high energy and with $1053 \mathrm{~nm}$ and $527 \mathrm{~nm}$ laser drive pulses. 
We attribute this turbulent feature in Xe to pre-ionization of the gas by the laser and likely beam filamentation of the pulse in the ionizing plasma. The most likely reason that the feature is seen on blast waves traveling through xenon gas and not on those traveling through nitrogen gas has to do with the different multi-photon ionization thresholds of these gasses. The threshold for multi-photon ionization in xenon is lower than in nitrogen. At $527 \mathrm{~nm}$ ionization is a 5 photon process in xenon, as compared to 7 in nitrogen, and the ratio increases for higher charge states. This means that there is likely more ionization and heating of the xenon gas caused by the laser's passage, creating a low gas density region. Therefore, the blast wave traveling over this area will pick up gas that is lower density and hotter than in other regions, causing it to slow down less as it travels, creating the bump like feature seen. In both our results and Grun et. al.'s ${ }^{9}$ the feature appears in the laser cone path, as is illustrated in the $1^{\text {st }}$ panel of Fig. 3. If the feature were associated with the Vishniac overstability, one would expect rippled features at all points on the spherical blast shell, depending on random noise in the background gas and the initial blast wave. The feature also appears independent of the blast wave velocity. In our shots the average velocity at early times (between $50 \mathrm{~ns}$ and $100 \mathrm{~ns}$ ) ranges from $\sim 12 \mathrm{~km} / \mathrm{s}$ for $10 \mathrm{~J}$ shots, below the $25 \mathrm{~km} / \mathrm{s}$ threshold predicted for the onset of the overstability in xenon gas predicted by Lamming ${ }^{12}$, to $\sim 140 \mathrm{~km} / \mathrm{s}$ for the 1000J shots, above this threshold. These facts support the idea that the feature is caused by the laser's passage. 


\section{Simulations}

We have performed simulations that support and further clarify the conclusions suggested by the data. We performed 2D simulations of blast wave evolution using the Lawrence Livermore National Laboratory code CALE ${ }^{17}$. CALE is a 2-D arbitrary Lagrangian Eulerian (ALE) code with a tabular equation of state (EOS) and interface tracking. For our simulations we assumed $\sim 7.5$ Torr of xenon gas for the initial background. A $0.5 \mathrm{~mm}$ Mo sphere was used as the target and the laser pulse was $100 \mathrm{~J}$ in $5 \mathrm{~ns}$, with a $0.4 \mathrm{~mm}$ focal spot size. Some results from the simulation are shown in Figure 4. The first panel of the figure shows a contour plot of the electron temperature $17 \mathrm{~ns}$ after the initial laser pulse. One can clearly see the hot plasma in the region of the laser cone. The second panel of the figure shows simulated density gradients of a blast wave traveling through 7.5 Torr of Xenon gas about $400 \mathrm{~ns}$ after the 100J drive beam struck the target. Finally the $3^{\text {rd }}$ panel shows experimental data taken at $\mathrm{t}=300 \mathrm{~ns}$ and $10 \mathrm{~J}$ drive. There is a strong similarity between the experimental result and the simulation. The perturbation on the blast wave's surface in the simulation is qualitatively similar to that seen in all our xenon experimental results and to that seen in previous experiments ${ }^{9}$.

\section{Conclusions}

In conclusion, we have performed a systematic examination of shockwaves traveling through 5-10 Torr of either xenon or nitrogen gas as a function of laser energy. Strongly radiative blast waves form at early time in $\mathrm{Xe}$, which produce a large radiative precursor ahead of the shock and which decelerate much faster than a Sedov energy conserving wave. We have also observed a striking feature that appears at all drive laser energies in 
xenon gas corresponding to the region where the drive laser had previously traveled.

This appears to result from a heating and ionization of the gas in this area by the laser which self filaments in this plasma. A warm, low density channel is created in which the blast wave will travel at higher velocities than in other regions of the gas. Our experimental results are supported by simulations. This result may explain the

experimental deviation with theoretical calculations seen in previous results ${ }^{9,12}$, where the maximal growth rate was seen at a different wavelength than expected and was larger than expected. The maximal growth rate feature appears to be produced by the laser's passage and is not a result of the Vishniac overstability. These results suggest that caution must be exercised when interpreting hydrodynamic effects in laser driven experiments in gas traversed by a laser pulse. There are several complicated interactions that can arise, especially a gas that is easily ionized, like xenon.

\section{Acknowledgements}

We would like to acknowledge helpful conversations with Keith Matzen and Bruce Remington. We would also like to thank the Lawrence Livermore National Laboratory for use of the Janus laser and the Sandia National Laboratory Inertial Confinement Fusion program for generous allocation of shot time on the Z-Beamlet laser. This work was supported by the US Dept. of Energy National Nuclear Security Agency under Cooperative agreement DE-FC52-03NA00156.

This work was performed under the auspices of the U. S. Department of Energy by University of California, Lawrence Livermore National Laboratory under contract No. W-7405-Eng-48. 


\section{Refrences}

1 J. J. Erpenbeck, Physics of Fluids 5 (10), 1181 (1962).

$2 \quad$ P.A. Isenberg, Astrophysical Journal 217 (2), 597 (1977).

3 G. L. Welter, Astronomy and Astrophysics 105 (2), 237 (1982); G. Welter and J. Schmidburgk, Astrophysical Journal 245 (3), 927 (1981).

$4 \quad$ C. F. Mckee and B. T. Draine, Science 252 (5004), 397 (1991); J. P. Ostriker and C. F. Mckee, Reviews of Modern Physics 60 (1), 1 (1988).

5 E. T. Vishniac, Astrophysical Journal 274 (1), 152 (1983); D. Ryu and E. T. Vishniac, Astrophysical Journal 368 (2), 411 (1991); D. Ryu and E. T. Vishniac, Astrophysical Journal 313 (2), 820 (1987). J. M. Blondin, E. B. Wright, K. J. Borkowski et al., Astrophysical Journal 500 (1), 342 (1998).

7 B. G. Elmegreen and D. M. Elmegreen, Astrophysical Journal 220 (3), 1051 (1978); B. G. Elmegreen and C. J. Lada, Astrophysical Journal 214 (3), 725 (1977).

$8 \quad$ M. M. MacLow and M. L. Norman, Astrophysical Journal 407 (1), 207 (1993). J. Grun, J. Stamper, C. Manka et al., Physical Review Letters 66 (21), 2738 (1991).

10 M. J. Edwards, A. J. MacKinnon, J. Zweiback et al., Physical Review Letters 8708 (8), art. no. (2001).

11 T. Ditmire, K. Shigemori, B. A. Remington et al., Astrophysical Journal Supplement Series 127 (2), 299 (2000); K. Shigemori, T. Ditmire, B. A.

Remington et al., Astrophysical Journal 533 (2), L159 (2000).

12 J. M. Laming and J. Grun, Physical Review Letters 89 (12) (2002); J. M. Laming and J. Grun, Physics of Plasmas 10 (5), 1614 (2003).

13 J.A. Glaze, W.W. Simmons, and W.F. Hagen, presented at the Proceedings of the Society of Photo-Optical Instrumentation Engineers, vol.76. Ultra High Power Lasers for Practicable Applications, Palos Verdes Estates, CA, USA

Reston, VA, USA, 1976 (unpublished).

15 I. A. B. Zel*dovich, I. U. P. Raizer, Wallace D. Hayes et al., Physics of shock waves and high-temperature hydrodynamic phenomena. (Academic Press, New York, 1966).

16 E. Cohen, T. Piran, and R. Sari, Astrophysical Journal 509 (2), 717 (1998).

17 CALE Users Manual. (Lawrence Livermore National Laboratory, 1991). 


\section{Figure Captions}

FIG. 1: Experimental setup of laser shock experiments. Drive beam, varying in energy from $10 \mathrm{~J}-1000 \mathrm{~J}$, enters the chamber from left and illuminates a pin target. This creates a blast wave that is imaged by the probe laser onto a dark field imaging telescope, shown in the inset. The dark field telescope blocks light that passes through the chamber undeflected, but any light that encounters a density gradient is deflected around the beam block at the center of the telescope and shows up as a bright area.

Figure 2: Blast wave trajectory through xenon and nitrogen gas. After $\sim 200$ ns in xenon the trajectory changes from $\mathrm{t}^{0.25}$, indicative of a highly radiative blast wave, to $\mathrm{t}^{0.4}$, consistent with the energy conserving Taylor-Sedov solution. The nitrogen blast wave trajectory is always consistent with the Taylor-Sedov solution.

Figure 3: Dark-field images of blast waves traveling through xenon (left) and nitrogen (right) gas produced by various drive laser energies. In all cases drive laser enters from left. From top to bottom drive laser energies are $\sim 10 \mathrm{~J}, \sim 500 \mathrm{~J}$, and $\sim 1000 \mathrm{~J}$. There is a contrast both in the small scale structure and the laser-side feature between the gasses.

Figure 4: The leftmost panel has a simulation of the electron temperature $17 \mathrm{~ns}$ after a $100 \mathrm{~J}$ laser pulse struck a pin target. The right two panels are comparison of simulated and experimental blast wave shapes. The simulation is $400 \mathrm{~ns}$ after a $100 \mathrm{~J}$ laser pulse. The experiment shows a blast wave $300 \mathrm{~ns}$ after a $10 \mathrm{~J}$ shot. 


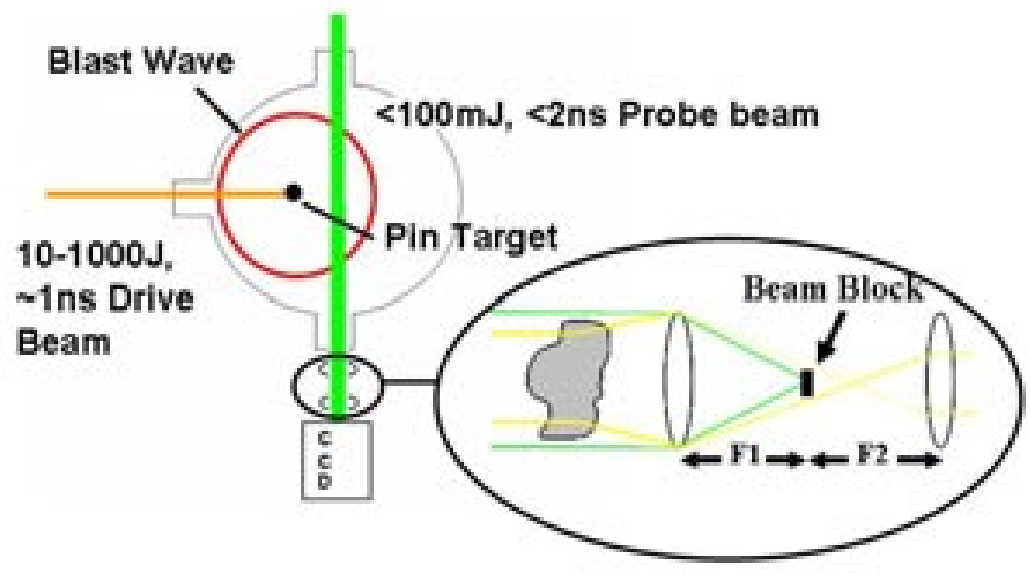



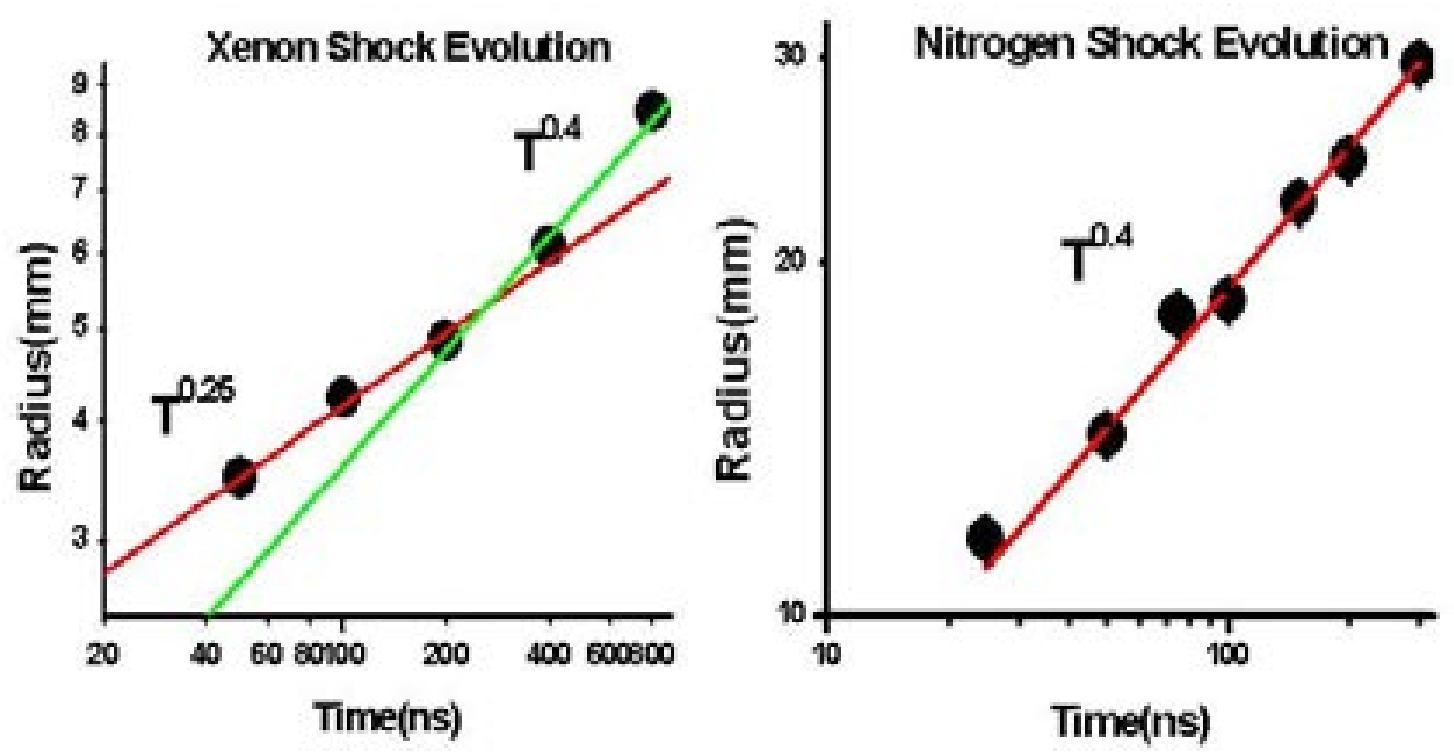


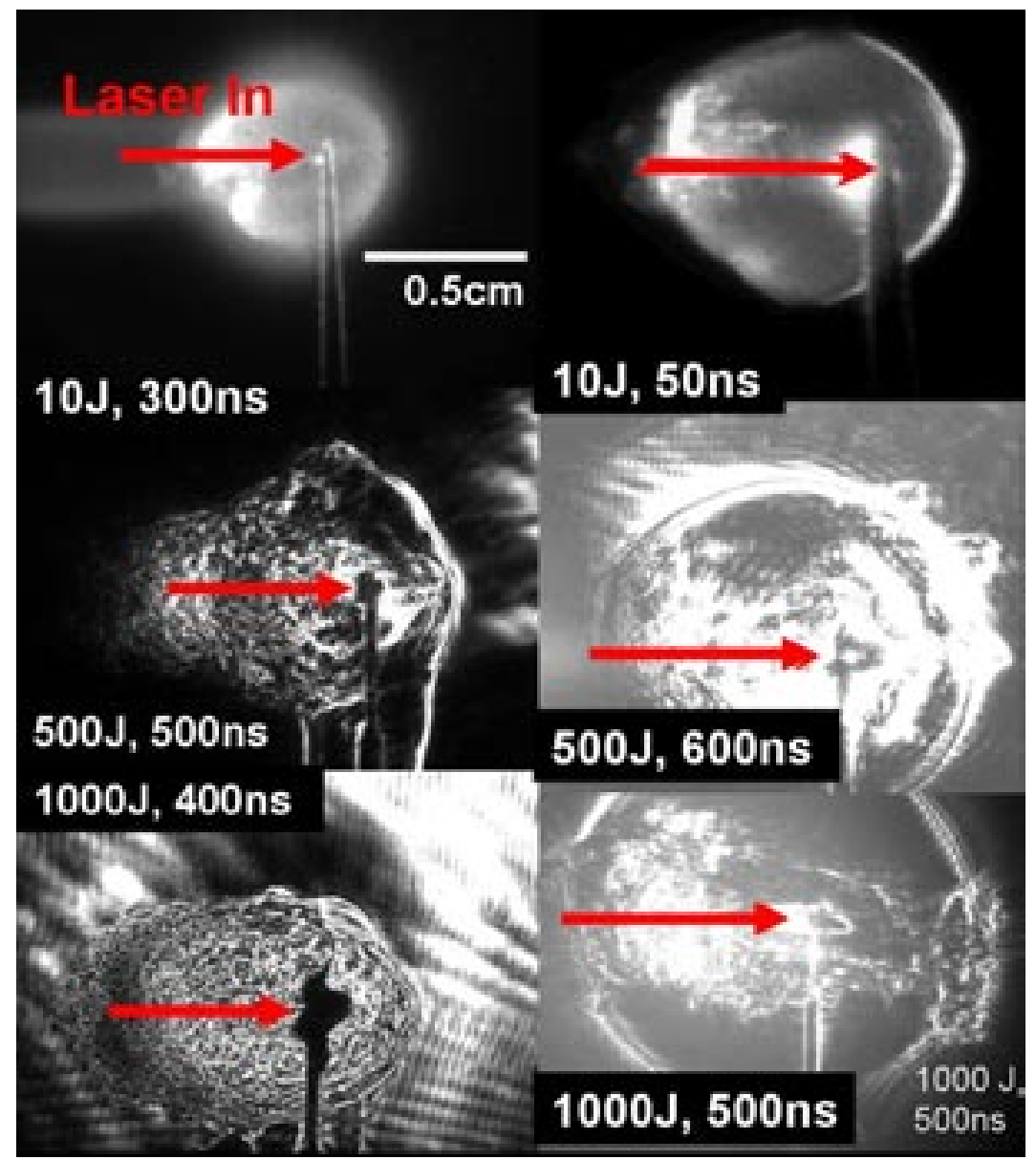


a

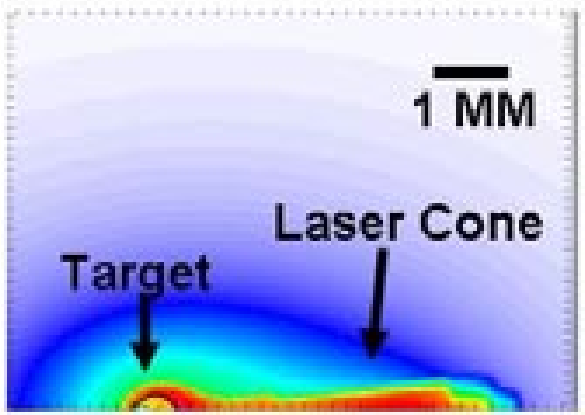

Experiment

Simulation

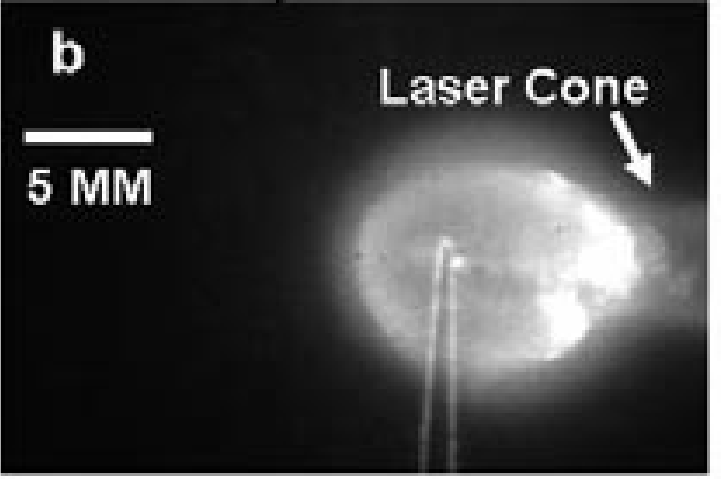

